\title{
Effect of gestational oily fish intake on the risk of allergy in children may be influenced by FADS1/2, ELOVL5 expression and DNA methylation
}

Purevsuren Losol ${ }^{1,2}$, Faisal I. Rezwann ${ }^{1}$, Veeresh K. Patil ${ }^{3,4}$, Carina Venter ${ }^{3}$, Susan Ewart ${ }^{5}$, Hongmei Zhang ${ }^{6}$,

S. Hasan Arshad ${ }^{3,4}$, Wilfried Karmaus ${ }^{6}$ and John W. Holloway ${ }^{1,4^{*}}$ (i)

\begin{abstract}
Background: Evidence suggests that prenatal exposure to $n-3$ long-chain polyunsaturated fatty acids (LCPUFA) reduces the incidence of allergic disease in children. LCPUFAs are produced from dietary precursors catalyzed by desaturases and elongases encoded by the FADS1/2 and ELOVL5 genes. DNA methylation regulates gene activity and fatty acid supplementation could alter DNA methylation (DNA-M) at these genes. We investigated whether DNA-M and expression of the FADS1/2 and ELOVL5 genes were associated with allergy in children and gestational fish intake. We studied 170 participants from the Isle of Wight 3rd Generation Cohort, UK. Phenotype data and exposure was assessed by questionnaires. Genome-wide DNA-M in cord blood samples was quantified using the Illumina Infinium HumanMethylation450 and EPIC Beadchips. Five SNPs (single-nucleotide polymorphisms) in the FADS gene cluster and one SNP in ELOVL5 were genotyped in offspring. FADS gene expression in offspring cord blood was determined.
\end{abstract}

Results: Gestational fish intake was significantly associated with increased methylation of $\operatorname{cg} 12517394(P=0.049)$, which positively correlated with FADS1 mRNA levels $(P=0.021)$. ELOVL5 rs2397142 was significantly associated with eczema $(P=0.011)$ and methylation at $c g 11748354$ and $\operatorname{cg} 24524396(P<0.001$ and $P=0.036$, respectively).

Gestational fish intake was strongly associated with elevated DNA-M at cg11748354 and cg24524396 $(P=0.029$ and $P=0.002$, respectively) and reduced ELOVL5 mRNA expression ( $P=0.028)$.

Conclusion: The association between induced FADS1/2 and ELOVL5 DNA-M and reduced gene expression due to gestational fish intake provide a mechanistic explanation of the previously observed association between maternal LCPUFA intake and allergy development in early childhood.

Keywords: Allergy, FADS, ELOVL, Fish intake, DNA methylation, Pregnancy

\section{Background}

Maternal diet during pregnancy is a potentially important determinant of intrauterine development and linked to fetal physiologic adaptations and early immune system programming [1]. To maintain fetal development, nutrients are transported to the fetus across the placenta

\footnotetext{
* Correspondence: j.w.holloway@soton.ac.uk

${ }^{1}$ Human Development and Health, Faculty of Medicine, University of Southampton, Southampton, UK

${ }^{4}$ Clinical and Experimental Sciences, Faculty of Medicine, University of Southampton, Southampton, UK

Full list of author information is available at the end of the article
}

[2]. Exposure to omega-6 (n-6) and omega-3 (n-3) polyunsaturated fatty acids (PUFAs) during pregnancy has been found to be associated with allergic outcomes in infants or children [3]. Maternal PUFA supplementation during pregnancy has been reported to reduce childhood asthma at age 16 [4] and to reduce the absolute risk of persistent wheeze or asthma and airway inflammation in offspring at 36 months after birth [5]. Oily fish consumption, a major source of $n-3$ PUFAs, contains higher amount of eicosapentaenoic acid (EPA) and docosahexaenoic acid (DHA) which has been shown to be

(c) The Author(s). 2019 Open Access This article is distributed under the terms of the Creative Commons Attribution 4.0 International License (http://creativecommons.org/licenses/by/4.0/), which permits unrestricted use, distribution, and 
protective against allergic diseases [6]. Fish intake has also been demonstrated to diminish the risk of asthma at 18 months [7]. In contrast, maternal shellfish consumption during the first trimester has been shown to increase the risk of wheezing, eczema and food allergy, while fatty fish consumption has been associated with increased risk of eczema in offspring $[8,9]$. However, a recent large prospective study could not substantiate the previously observed beneficial association between fish and seafood consumption in pregnancy and development of asthma and allergic rhinitis symptoms in children up to 8 years of age [10]. In contrast, a metaanalysis of six studies revealed an association between fish oil supplementation during pregnancy and reduced risk of sensitization to food allergens at first year [11].

Long-chain PUFAs (LCPUFAs), produced from their dietary precursors (omega-3 and omega-6), are catalyzed by desaturases and elongases which are encoded by the $F A D S 1 / 2$ and ELOVL2/5 genes. A number of studies have found an association between FADS gene variants and immune-related outcomes. For example, maternal FADS genetic variation, through a higher infant supply of LCPUFA, has been found to be associated with a decrease in the production of IL-5, IL-10, and IL-17 in the infant [12-16]. Carriers of the minor alleles of FADS genetic variants and their respective haplotypes have been demonstrated to have lower levels of desaturase products and a lower prevalence of allergic rhinitis and atopic eczema [16]. An observational study showed that the FADS SNP rs3834458 was significantly associated with serum LCPUFA levels, with individuals homozygous for the del/ del variant shown to have a decreased level of arachidonic acid (AA) and increased alpha-linolenic acid (ALA) to DHA ratio in high fish-eating mothers [17]. Previously, it has been reported that carriers of the minor alleles of FADS SNPs, including rs3834458, tend to have a lower blood composition of LCPUFA, particularly AA [18]. Although the evidence is not conclusive, it is conceivable that minor alleles of FADS genes produce a lower proportion of desaturase products and therefore less AA, reducing the risk of asthma.

Increased DNA methylation (DNA-M) at FADS promoter regions was associated with lower gene expression levels in minor homozygote carriers [19], and differences in DNA-M associated with the development of asthma during childhood has been reported [20, 21]. There are suggestions that DNA-M may regulate FADS activity [22] and that fatty acid supplementation can induce altered methylation of specific CpG loci in FADS2 and ELOVL5 [23]. A murine study reported differing levels of $F A D S 2$ promoter methylation in the liver tissue from offspring exposed to linoleic acid during gestation [24]. Recently, allele-specific methylation was reported between rs174537 and DNA methylation in FADS region in leukocyte and CD4+ cells [25].
Given these interrelations between maternal diet, genotype, offspring DNA-M, and asthma, we hypothesized that maternal fish intake may modulate offspring epigenetic programming, regulating fatty acid desaturase and elongase activities, and that this may modulate later health outcomes, in particular childhood wheeze and eczema.

\section{Results}

The descriptive characteristics of the cohort are presented in Table 1. There were no substantial differences in the prevalence of maternal smoking during pregnancy, maternal history of asthma, maternal history of eczema, maternal socioeconomic status, child eczema, child wheeze, and maternal oily fish intake between the total cohort and those who were randomly selected for the DNA-methylation analysis (Table 1). For each FADS and ELOVL5 association analysis, 170 methylationphenotype pairs, 157 methylation-gene expression pairs and phenotype-gene expression pairs, 123 methylationgenotype, phenotype-genotype, and gene expressiongenotype pairs were available (Fig. 1).

Three FADS1 and FADS2 SNPs (rs174537, rs174556, rs174575) and one ELOVL5 SNP (rs2397142) were genotyped and used for further analysis as these were not in linkage disequilibrium $\left(r^{2} \leq 0.80\right)$. Associations of these SNPs with the prevalence of wheeze and eczema were assessed as shown in Table 2. Among these, the ELOVL5 variant rs2397142 was associated with eczema $(P=0.011)$. The eczema prevalence was higher in minor allele carriers than in homozygous major allele carriers.

A total of 39 CpG sites spanning the genomic region of the FADS cluster and $27 \mathrm{CpG}$ sites in ELOVL5 were analyzed for association with wheeze and eczema. Methylation levels of $\operatorname{cg} 00786201$ and $\operatorname{cg} 25448062$ in the FADS cluster showed an association with eczema, and methylation at $\operatorname{cg} 01400685$ was found to be associated with wheeze $(P<0.05$, respectively; Additional file 1$)$. Also, methylation levels of cg18564099 in ELOVL5 showed an association with eczema ( $P=0.046$; Additional file 1$)$.

Maternal oily fish intake was correlated with significantly increased DNA-M level at cg12517394 in FADS $(P=0.049$, Table 3$)$.

In addition, maternal oily fish intake was strongly associated with elevated DNA-M at cg11748354 and cg24524396 in ELOVL5 $(P=0.029$ and $P=0.002$, respectively, Table 4) and reduced ELOVL5 mRNA expression $(P=0.028$, Table 5$)$.

A genotype-dependent interaction was identified between ELOVL5 SNPs and CpG sites $(\operatorname{cg} 11748354 \times$ rs2397142 and $\operatorname{cg} 24524396 \times \operatorname{rs} 2397142(P<0.001$ and $P=0.036$, respectively; Additional file 2). When the correlation between methylation levels and gene expression levels in cord blood were tested, DNA-M at cg12517394 was significantly correlated with FADS1 gene expression 
Table 1 Characteristics of subjects with available methylation data compared to the participants of the total

\begin{tabular}{|c|c|c|c|}
\hline & $\begin{array}{l}\text { Total participants } \\
n=436(\%)\end{array}$ & $\begin{array}{l}\text { Participants with } \\
\text { DNA-M data } \\
n=170(\%)\end{array}$ & $P$ value \\
\hline \multicolumn{4}{|c|}{ Maternal smoking during pregnancy } \\
\hline Yes & $146(36.6)$ & $61(37.0)$ & 0.999 \\
\hline No & $253(63.4)$ & $104(63.0)$ & \\
\hline \multicolumn{4}{|l|}{ Maternal history of asthma } \\
\hline Yes & $64(18.4)$ & $27(18.2)$ & $\overrightarrow{0.999}$ \\
\hline No & $284(81.6)$ & $121(81.8)$ & \\
\hline Missing & 88 & 22 & \\
\hline \multicolumn{4}{|l|}{ Maternal history of eczema } \\
\hline Yes & $143(41.6)$ & $65(40.4)$ & 0.846 \\
\hline No & $201(58.4)$ & $96(59.6)$ & \\
\hline NA & 92 & 9 & \\
\hline \multicolumn{4}{|l|}{ Child eczema } \\
\hline Yes & $58(15.8)$ & $29(18.7)$ & 0.442 \\
\hline No & $308(84.2)$ & $126(81.3)$ & \\
\hline Missing & 70 & 15 & \\
\hline \multicolumn{4}{|l|}{ Child wheeze } \\
\hline Yes & $67(18.4)$ & $32(20.6)$ & 0.625 \\
\hline No & $298(81.6)$ & $123(79.4)$ & \\
\hline Missing & 71 & 15 & \\
\hline \multicolumn{4}{|l|}{ Maternal fish intake } \\
\hline Never & $159(61.9)$ & $70(61.4)$ & 0.801 \\
\hline $1-2$ or less per month & $74(28.8)$ & $32(28.1)$ & \\
\hline 1-3 per week & $21(8.2)$ & $11(9.6)$ & \\
\hline $\begin{array}{l}4 \text { or more times per } \\
\text { week }\end{array}$ & $2(0.8)$ & $0(0)$ & \\
\hline Uncertain & $1(0.4)$ & $1(0.9)$ & \\
\hline Missing & 179 & 52 & \\
\hline \multicolumn{4}{|c|}{ Maternal socioeconomic status } \\
\hline Below low & 77 (19.3) & $25(15.1)$ & 0.273 \\
\hline Low & $97(24.3)$ & $54(32.5)$ & \\
\hline Medium Low & $114(28.5)$ & $48(28.3)$ & \\
\hline Medium & $61(15.3)$ & $24(14.5)$ & \\
\hline High & $51(12.8)$ & $16(9.6)$ & \\
\hline NA & 36 & 3 & \\
\hline
\end{tabular}

Data are $n(\%)$, unless otherwise indicated. Difference between groups was assessed using Yates' continuity correction DNA-M DNA methylation, NA not applicable

levels $(P=0.021$, Additional file 3$)$. FADS1 gene expression levels were significantly different according to the FADS SNPs $(P<0.05$, Additional file 4$)$, but not in FADS2 and ELOVL5.

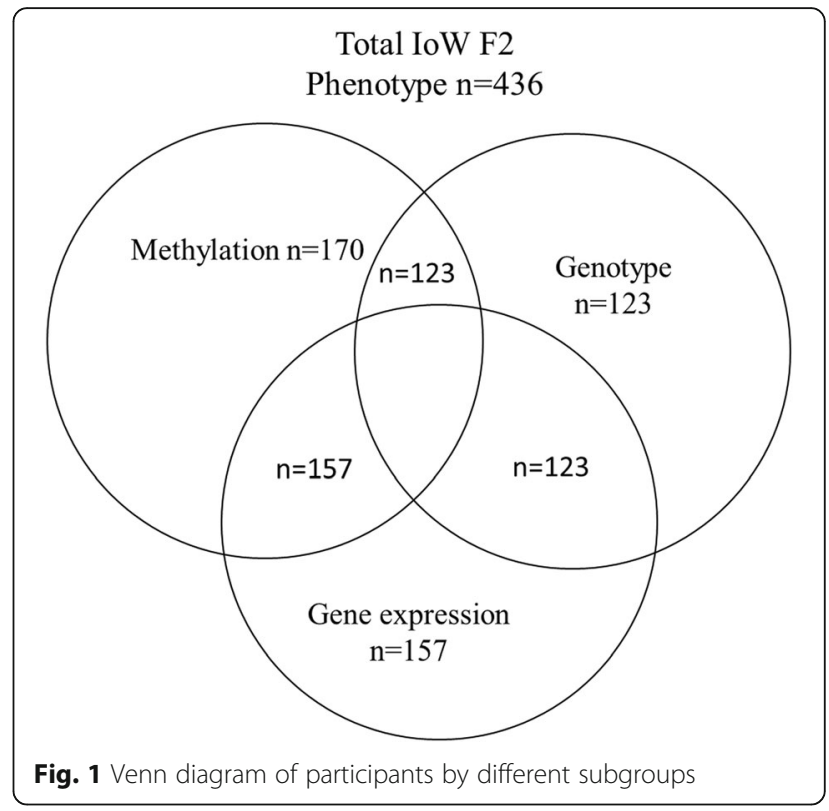

\section{Discussion}

This study investigated the effects of oily fish intake during the third trimester of pregnancy on the allergic outcomes of the infants by using a subset of umbilical cord blood samples from Isle of Wight (IoW) $\mathrm{F}_{2}$ generation. This is the first study to explore associations between FADS and ELOVL SNPs, gene expression, DNA methylation, child allergy, and gestational oily fish intake in the same cohort. Maternal fish intake could increase mean maternal circulating omega-3 levels and increased cord blood fatty acids that potentially prevent inflammation [26].

Our first major finding was a significantly increased cord blood methylation of cg12517394 in the FADS cluster and DNA methylation at ELOVL5, cg11748354, and cg24524396 with gestational oily fish intake, whereas oily fish intake during pregnancy reduced ELOVL5 mRNA expression in the cord blood. In previous studies, supplementation with $n$-3 LCPUFA was shown to increase methylation levels at CpGs in FADS2 and ELOVL5, which were negatively associated with the level of the FADS2 and ELOVL5 transcripts in non-atopic adults consuming $n$-3 LCPUFA for 8-12 weeks [23]. In contrast, it was reported that maternal fish oil supplementation during the second half of pregnancy had small or no effects on DNA-M of infants $[27,28]$. In vivo, feeding rats a fish oil-enriched diet for 9 weeks induced lower FADS2 mRNA expression and increased methylation of specific CpG loci in FADS2 [29]. Together, these findings suggest that methylation at these loci directly regulates FADS and ELOVL5 transcription, and $n-3$ LCPUFA intake can induce changes in methylation levels in specific genes. 
Table 2 Prevalence of wheeze and eczema in offspring stratified by genotype

\begin{tabular}{|c|c|c|c|c|c|c|}
\hline Gene & SNPS & Genotypes & $\begin{array}{l}\text { Wheeze ever } \\
n(\%)\end{array}$ & $P$ value & $\begin{array}{l}\text { Eczema ever } \\
n(\%)\end{array}$ & $P$ value \\
\hline \multirow[t]{3}{*}{ FADS1 } & rs174537 & GG & $11(21.6)$ & $>0.999$ & 10 (19.6) & 0.849 \\
\hline & & GT & $11(22.0)$ & & $12(23.5)$ & \\
\hline & & $\Pi$ & $3(25.0)$ & & $2(16.7)$ & \\
\hline \multirow[t]{3}{*}{ FADS1 } & rs174556 & CC & $11(20.0)$ & 0.669 & $11(20.0)$ & 0.939 \\
\hline & & CT & $11(24.4)$ & & $11(23.9)$ & \\
\hline & & $\pi$ & $1(12.5)$ & & $2(25.0)$ & \\
\hline \multirow[t]{3}{*}{ FADS2 } & rs174575 & CC & $12(19.4)$ & 0.614 & $12(19.4)$ & 0.939 \\
\hline & & $C G$ & $10(23.8)$ & & $10(23.3)$ & \\
\hline & & GG & $3(33.3)$ & & $2(22.2)$ & \\
\hline \multirow[t]{3}{*}{ ELOVL5 } & rs2397142 & $C C$ & $9(20.0)$ & 0.569 & $4(8.9)$ & 0.011 \\
\hline & & $C G$ & $14(25.9)$ & & $18(32.7)$ & \\
\hline & & GG & $2(14.3)$ & & $2(14.3)$ & \\
\hline
\end{tabular}

Data are $n$ (\%). Chi-square test was applied with Yates' correction

Our second major finding was a genotype-dependent methylation of cg11748354 and cg24524396 related to ELOVL5 rs2397142. A previous meta-analysis in liver methylation data, a highly relevant tissue for PUFA metabolism, identified a strong effect of $F A D S$ rs174537 on the methylation status of one or more critical CpG sites in the FADS gene cluster [19]. The most significant association was observed with cg27386326 $\left(p=2.69 \times 10^{-29}\right)$ and four other sites, including cg16213375, cg10515671, cg03805684, and cg19610905 [22]. We did not observe genotype-dependent methylation of FADS1. However, we identified higher gene expression levels for FADS1 in the cord blood with positive correlation with methylation at cg12517394 that are likely to be influenced by FADS genetic variants.

Finally, rs2397142 in ELOVL5 showed significant association with eczema in this study. The frequency of GG genotype of this polymorphism was higher in children with eczema. In a previous study, children from mothers with low fatty fish consumption during pregnancy had higher risk of eczema [8]. Furthermore, children with atopic eczema had lower ELOVL5 mRNA levels in their blood when compared to healthy controls [30]. These findings indicate that maternal lower fatty acid levels during pregnancy directly associated with decreased

Table 3 Association of maternal fish intake with cord blood FADS DNA methylation

\begin{tabular}{llll}
\hline CpG & \multicolumn{2}{l}{ Oily fish intake (28 weeks) } & $P$ value \\
\cline { 2 - 3 } & No $(n=103)$ & Yes $(n=11)$ & \\
\hline cg00614641 & $0.088 \pm 0.013$ & $0.079 \pm 0.010$ & 0.022 \\
cg07999042 & $0.880 \pm 0.013$ & $0.870 \pm 0.020$ & 0.033 \\
cg12517394 & $0.037 \pm 0.007$ & $0.040 \pm 0.005$ & 0.049 \\
\hline
\end{tabular}

Data are mean (SD). $P$ values calculated using the Mann-Whitney $U$ test
ELOVL5 mRNA expression in children and increase the risk of eczema, especially in children carrying GG genotype of rs2397142.

Our study had some limitations. Data on DNA-M and gene expression were only available from the cord blood and not from the liver samples, the major site of fatty acid metabolism. In addition, only proxy measures of maternal LCPUFA intake in the form of self-reported oily fish intake were available. We were unable to determine the ration of omega- 6 to omega- 3 in this cohort. This has previously been indicated as a possible risk factor for the development of atopic dermatitis and allergic rhinitis [31] and should be addressed in future studies. Nonetheless, the SNP-DNA-M gene expression associations observed largely support previous observations. Finally, this study was underpowered to investigate weaker links between maternal oily fish intake and clinical outcomes in children; however, there was a trend for a lower incidence of early life wheeze in the group whose mothers consumed oily fish compared with those who did not.

\section{Conclusions}

We report that infants of mothers supplemented with higher oily fish intake during the last trimester of

Table 4 Association of maternal fish intake with cord blood ELOVL5 DNA methylation

\begin{tabular}{llll}
\hline CpG & \multicolumn{2}{l}{ Oily fish intake $(28$ weeks $)$} & $P$ value \\
\cline { 2 - 3 } & No $(n=103)$ & Yes $(n=11)$ & \\
\hline cg10410213 & $0.608 \pm 0.025$ & $0.079 \pm 0.020$ & 0.010 \\
cg11748354 & $0.146 \pm 0.045$ & $0.191 \pm 0.073$ & 0.029 \\
cg24524396 & $0.093 \pm 0.011$ & $0.106 \pm 0.012$ & 0.002 \\
\hline
\end{tabular}

Data are mean (SD). $P$ values calculated using the Mann-Whitney $U$ test 
Table 5 Gestational oily fish intake on offspring gene expression

\begin{tabular}{llll}
\hline & \multicolumn{2}{l}{ Oily fish intake $(28$ weeks $)$} & $P$ value \\
\cline { 2 - 3 } & No $(n=81)$ & Yes $(n=9)$ & \\
\hline FADS1 mRNA & $6.77 \pm 0.42$ & $6.68 \pm 0.52$ & 0.691 \\
FADS2 mRNA & $12.13 \pm 0.80$ & $12.66 \pm 0.90$ & 0.090 \\
Intergenic mRNA & $8.57 \pm 0.59$ & $8.95 \pm 0.62$ & 0.104 \\
ELOVL5 mRNA & $8.47 \pm 0.43$ & $7.93 \pm 0.63$ & 0.028 \\
\hline
\end{tabular}

Data are mean (SD). $P$ values calculated using the Mann-Whitney $U$ test

pregnancy had greater methylation levels at FADS gene cluster and ELOVL5 and lower levels of ELOVL5 mRNA expression. These methylation and gene expression changes were modulated by FADS and ELOVL gene variants. In conclusion, the association between induced FADS and ELOVL DNA methylation and reduced gene expression due to gestational fish intake gives a mechanistic explanation of previously observed associations between maternal LCPUFA intake and allergy development in early childhood.

\section{Methods}

\section{Study population}

Children born on the Isle of Wight (IoW) between 1989 and $1990 \quad(n=1536)$ were recruited to prospectively study the natural history of asthma, allergy, and obesity. Informed consent was obtained from the parents (1st generation, IoW $\mathrm{F}_{0}$ ), and after exclusion, 1456 participants were recruited (2nd generation, IoW $F_{1}$ ) as the IoW birth cohort [32]. The recruitment of newborns for the IoW 3rd Generation ( $\mathrm{IoW} \mathrm{F}_{2}$ ) study started in April 2010, and the samples used in this study are from infants born between April 2010 and May 2018. In total, 436 newborns have been recruited such that at least one of their parents is in the IoW $F_{1}$ [33]. In this study, we have used cord blood samples from a subset of IoW $F_{2}$ generation.

\section{Clinical data collection}

In the $F_{2}$ generation, maternal history of asthma and smoking during pregnancy was ascertained at birth. Early wheezing was defined if symptoms of "wheeze" were reported by parents to occur between cold/infections at least once at either the 3-, 6-, or 12-month follow-up after birth. Childhood eczema information was also collected at 3, 6, and 12 months and defined as chronic or chronically relapsing, itchy dermatitis lasting more than six weeks with characteristic morphology and distribution $[34,35]$.

\section{Assessment of fish and shellfish intake}

Participant's mothers were asked to fill out a validated food frequency questionnaire that inquired about their usual consumption, before pregnancy, during 24th and 28th week of gestation [36]. White fish, shellfish, and oily fish consumption was evaluated with women reporting their frequency of consumption on a 5-point scale: "never," "rarely (1-2 or less per month)," "occasionally (1-3 per week)," "4 or more times per week," and "uncertain." Mackerel, salmon, sardines, pilchards, herring, kipper, whitebait, trout, crab, and fresh tuna were classified as oily fishes. We considered oily fish intake only in the current study because of its high omega-3 contents.

\section{SNP selection for FADS1, FADS2, and ELOVL5 genes}

Five candidate SNPs-FADS1 (Entrez Gene 3992) rs174537, rs174545, and rs174556; intergenic between FADS1-FADS2 (Entrez Gene 9415) rs3834458; and FADS2 rs174575 and ELOVL5 rs2397142-were selected based on evidence of association with LCPUFA proportions in human plasma, tissues, and milk [37], or because the SNPs had been suggested to play an important role in regulation of FADS1/2 activity because of their location near potential regulatory regions [16]. Three of the five SNPs (rs174537, rs174545, and rs3834458) are from the same haplotype block $\left(r^{2}>0.80\right)$. Therefore, in our analysis, we have only used four unrelated SNPs: rs174537, rs174556, rs174575, and rs2397142. For the IoW $F_{2}$, genotyping was conducted using cord blood DNA samples $(n=123)$ with the genome-wide Infinium OmniExpressExome-8 Kit, and all six SNPs were imputed using the 1000 genome reference panel [38].

\section{DNA methylation}

DNA was extracted from the cord blood samples of IoW $\mathrm{F}_{2}$ over six batches, using a standard salting out procedure [39]. The DNA concentration was determined by PicoGreen quantitation. One microgram of DNA was bisulfite-treated for cytosine to thymine conversion using the EZ 96-DNA methylation kit (Zymo Research, Irvine, CA, USA), following the manufacturer's standard protocol. Genome-wide DNA-M was assessed in 130 samples using the Illumina Infinium HumanMethylation450 BeadChip (Illumina, Inc., San Diego, CA, USA) and using the Illumina Infinium MethylationEPIC Beadchip array for 63 samples. Arrays were processed using a standard protocol as previously described [40], with multiple identical control samples assigned to each bisulphite conversion batch to assess assay variability, and samples were randomly distributed on microarrays to control against batch effects. The degree of DNA methylation at each CpG site was recorded as beta value, ranging from 0 (no methylation) to 1 (completely methylated). One sample was excluded due to maternal blood contamination. The CPACOR pipeline [41] was used to pre-process the methylation $450 \mathrm{k}$ and EPIC data for quality control, and batch correction was done using ComBat [42]. In the final analysis, 170 samples were used with 
complete methylation information. The methylation levels of 39 CpG sites spanning the genomic region of the FADS cluster and $27 \mathrm{CpGs}$ of the ELOVL5 were selected.

\section{Gene expression}

FADS and ELOVL5 gene expression in the cord blood $(n=157)$ was determined by total RNA extracted from the cord blood using SurePrint G3 Human Gene Expression Microarrays (GeneSpring Technology 39,494) [43]. Microarray data was processed using limma [43] in the $\mathrm{R}$ statistical computing environment, and background correction was performed using normal-exponential convolution (normexp) function [44]. Data was converted to $\log 2$-transformed data for further analysis. Filtering was performed to remove low expressed probes that are close to the background level. Negative control probes were also removed from the data.

\section{Statistical analysis}

To assess whether our analytic sample (170 DNA samples) was representative of the total cohort available $(n=436)$, we compared the characteristics of the two subsets using chi-squared tests. Distribution and prevalence of wheeze and eczema according to genotype were compared using the $\chi^{2}$ test with Yates' continuity correction. The genotype frequency of candidate SNPs was examined for a significant departure from the Hardy-Weinberg equilibrium using a $\chi^{2}$ test. The methylation levels of the FADS cluster and ELOVL5 were tested for association with wheeze and eczema up to 1 year using Mann-Whitney $U$ tests to compare independent samples. SNP genotypedependent methylation and genotype-dependent gene expression were analyzed using the Kruskal-Wallis non-parametric test to determine the difference between three genotype groups on continuous variables. A Spearman correlation test was used to analyze the relationship between DNA methylation levels and gene expression. Comparison of DNA-M and gene expression levels between oily fish intake status at 28 gestational weeks were tested by the non-parametric two-sample Mann-Whitney $U$ test. Bonferroni correction was not undertaken as these were a priori hypothesis instead of a random set of CpGs and genotypes. Under this situation, multiple testing adjustments may not be necessary as suggested by Rothman [45].

All statistical analyses were performed using IBM SPSS Statistics, Version 21.0 (IBM Corp., Armonk, NY, USA), except for preprocessing of DNA methylation data, which was done using R statistical package [41]. The $P$ values of $<0.05$ were considered to indicate significance.

\section{Additional files}

Additional file 1: Association of wheeze and eczema with cord blood DNA methylation. (XLSX 9 kb)

Additional file 2: Genotype-dependent methylation. (XLSX 9 kb) Additional file 3: Change in relative mRNA expression compared to change in the methylation status of FADS1 methylation cg12517394. (XLSX $8 \mathrm{~kb}$ )

Additional file 4: Gene expression according to the FADS genotypes. (XLSX $9 \mathrm{~kb}$ )

\section{Abbreviations}

AA: Arachidonic acid; ALA: a-Linolenic acid; DHA: Docosahexaenoic acid; DNA-M: DNA methylation; ELOVL: Fatty acid elongase; EPA: Eicosapentaenoic acid; FADS: Fatty acid desaturase; IL: Interleukin; loW: Isle of Wight;

LCPUFA: Long-chain polyunsaturated fatty acids; mRNA: Messenger RNA; SNP: Single-nucleotide polymorphism

\section{Acknowledgements}

Not applicable

\section{Authors' contributions}

$\mathrm{PL}, \mathrm{JWH}, \mathrm{SHA}$, and $\mathrm{WK}$ formulated the research questions and designed the study. SHA, VKP, CV, and SE generated the data. PL, FIR, and $\mathrm{HZ}$ analyzed the data. All authors read and approved the final manuscript.

\section{Funding}

This study was funded by the National Institutes of Health (R01Al091905, R01HL132321). The 18-year follow-up of the Isle of Wight Birth Cohort was funded by NIH/NHLBI R01 HL082925-01 (PI: S. Hasan Arshad). Purevsuren Losol was the recipient of a long-term research fellowship of the European Respiratory Society (2015).

\section{Availability of data and materials}

The datasets used and/or analyzed during the current study are available from the corresponding author on reasonable request.

Ethics approval and consent to participate

Ethical approvals were obtained from Local Research Ethics Committees (last: NRES Committee South Central-Hampshire B).

Consent for publication

Not applicable

\section{Competing interests}

The authors declare that they have no competing interests.

\section{Author details}

${ }^{1}$ Human Development and Health, Faculty of Medicine, University of Southampton, Southampton, UK. ${ }^{2}$ Department of Molecular Biology and Genetics, School of Biomedicine, Mongolian National University of Medical Sciences, Ulaanbaatar, Mongolia. ${ }^{3}$ The David Hide Asthma and Allergy Research Centre, Isle of Wight, UK. ${ }^{4}$ Clinical and Experimental Sciences, Faculty of Medicine, University of Southampton, Southampton, UK. ${ }^{5}$ Department of Large Animal Clinical Sciences, Michigan State University, East Lansing, MI, USA. ${ }^{6}$ Division of Epidemiology, Biostatistics, and Environmental Health, School of Public Health, University of Memphis, Memphis, TN, USA.

Received: 18 December 2018 Accepted: 5 June 2019

Published online: 18 June 2019

\section{References}

1. Lockett GA, Huoman J, Holloway JW. Does allergy begin in utero? Pediatr Allergy Immunol. 2015;26(5):394-402.

2. Brett KE, Ferraro ZM, Yockell-Lelievre J, Gruslin A, Adamo KB. Maternal-fetal nutrient transport in pregnancy pathologies: the role of the placenta. Int Mol Sci. 2014;15(9):16153-85. 
3. Miles EA, Calder PC. Omega- 6 and omega-3 polyunsaturated fatty acids and allergic diseases in infancy and childhood. Curr Pharm Des. 2014;20(6):946-53.

4. Olsen SF, Østerdal ML, Salvig JD, Mortensen LM, Rytter D, Secher NJ, et al. Fish oil intake compared with olive oil intake in late pregnancy and asthma in the offspring: 16 y of registry-based follow-up from a randomized controlled trial. Am J Clin Nutr. 2008:88(1):167-75.

5. Bisgaard H, Stokholm J, Chawes BL, Vissing NH, Bjarnadóttir E, Schoos AM, et al. Fish oil-derived fatty acids in pregnancy and wheeze and asthma in offspring. N Engl J Med. 2016;375(26):2530-9.

6. Miles EA, Calder PC. Can early omega-3 fatty acid exposure reduce risk of childhood allergic disease? Nutrients. 2017;9(7). pii: E784.

7. Maslova E, Strøm M, Oken E, Campos H, Lange C, Gold D, et al. Fish intake during pregnancy and the risk of child asthma and allergic rhinitis longitudinal evidence from the Danish National Birth Cohort. Br J Nutr. 2013;110(7):1313-25

8. Leermakers ET, Sonnenschein-van der Voort AM, Heppe DH, de Jongste JC, Moll HA, Franco OH, et al. Maternal fish consumption during pregnancy and risks of wheezing and eczema in childhood: the Generation R Study. Eur J Clin Nutr. 2013;67(4):353-9.

9. Pelé F, Bajeux E, Gendron H, Monfort C, Rouget F, Multigner L, et al. Maternal fish and shellfish consumption and wheeze, eczema and food allergy at age two: a prospective cohort study in Brittany, France. Environ Health. 2013;12:102.

10. Stratakis N, Roumeliotaki T, Oken E, Ballester F, Barros H, Basterrechea M, et al. Fish and seafood consumption during pregnancy and the risk of asthma and allergic rhinitis in childhood: a pooled analysis of 18 European and US birth cohorts. Int J Epidemiol. 2017;46(5):1465-77.

11. Garcia-Larsen V, lerodiakonou D, Jarrold K, Cunha S, Chivinge J, Robinson Z, et al. Diet during pregnancy and infancy and risk of allergic or autoimmune disease: a systematic review and meta-analysis. PLoS Med. 2018;15(2): e1002507.

12. Hester AG, Murphy RC, Uhlson CJ, Ivester P, Lee TC, Sergeant S, et al. Relationship between a common variant in the fatty acid desaturase (FADS) cluster and eicosanoid generation in humans. J Biol Chem. 2014;289(32): 22482-9.

13. Muc M, Kreiner-Møller E, Larsen JM, Birch S, Brix S, Bisgaard $H$, et al. Maternal fatty acid desaturase genotype correlates with infant immune responses at 6 months. Br J Nutr. 2015;114(6):891-8.

14. Standl M, Sausenthaler S, Lattka E, Koletzko S, Bauer CP, Wichmann HE, et al. FADS gene variants modulate the effect of dietary fatty acid intake on allergic diseases in children. Clin Exp Allergy. 2011;41(12):1757-66.

15. Standl M, Sausenthaler S, Lattka E, Koletzko S, Bauer CP, Wichmann HE, et al. FADS gene cluster modulates the effect of breastfeeding on asthma. Results from the GINIplus and LISAplus studies. Allergy. 2012;67(1):83-90.

16. Schaeffer $L$, Gohlke H, Müller M, Heid IM, Palmer LJ, Kompauer I, et al. Common genetic variants of the FADS1 FADS2 gene cluster and their reconstructed haplotypes are associated with the fatty acid composition in phospholipids. Hum Mol Genet. 2006;15(11):1745-56.

17. Yeates AJ, Love TM, Engström K, Mulhern MS, McSorley EM, Grzesik K, et al. Genetic variation in FADS genes is associated with maternal long-chain PUFA status but not with cognitive development of infants in a high fisheating observational study. Prostaglandins Leukot Essent Fatty Acids. 2015; 102-103:13-20.

18. Rzehak P, Heinrich J, Klopp N, Schaeffer L, Hoff S, Wolfram G, et al. Evidence for an association between genetic variants of the fatty acid desaturase 1 fatty acid desaturase 2 ( FADS1 FADS2) gene cluster and the fatty acid composition of erythrocyte membranes. Br J Nutr. 2009;101(1):20-6.

19. Rahbar E, Ainsworth HC, Howard TD, Hawkins GA, Ruczinski I, Mathias R, et al. Uncovering the DNA methylation landscape in key regulatory regions within the FADS cluster. PLoS One. 2017;12(9):e0180903.

20. Soto-Ramírez N, Arshad SH, Holloway JW, Zhang H, Schauberger E, Ewart S, et al. The interaction of genetic variants and DNA methylation of the interleukin-4 receptor gene increase the risk of asthma at age 18 years. Clin Epigenetics. 2013;5(1):1.

21. DeVries A, Vercelli D. Epigenetic mechanisms in asthma. Ann Am Thorac Soc. 2016;13(Suppl 1):S48-50

22. Howard TD, Mathias RA, Seeds MC, Herrington DM, Hixson JE, Shimmin LC, et al. DNA methylation in an enhancer region of the FADS cluster is associated with FADS activity in human liver. PLoS One. 2014;9(5):e97510.

23. Hoile SP, Clarke-Harris R, Huang RC, Calder PC, Mori TA, Beilin LJ, et al. Supplementation with $\mathrm{N}-3$ long-chain polyunsaturated fatty acids or olive oil in men and women with renal disease induces differential changes in the DNA methylation of FADS2 and ELOVL5 in peripheral blood mononuclear cells. PLoS One. 2014;9(10):e109896.

24. Niculescu MD, Lupu DS, Craciunescu CN. Perinatal manipulation of alphalinolenic acid intake induces epigenetic changes in maternal and offspring livers. FASEB J. 2013;27(1):350-8.

25. Rahbar E, Waits CMK, Kirby EH Jr, Miller LR, Ainsworth HC, Cui T, et al. Allelespecific methylation in the FADS genomic region in DNA from human saliva, CD4+ cells, and total leukocytes. Clin Epigenetics. 2018;10:46.

26. Greenberg JA, Bell SJ, Ausdal WV. Omega-3 fatty acid supplementation during pregnancy. Rev Obstet Gynecol. 2008;1(4):162-9.

27. van Dijk SJ, Zhou J, Peters TJ, Buckley M, Sutcliffe B, Oytam Y, et al. Effect of prenatal DHA supplementation on the infant epigenome: results from a randomized controlled trial. Clin Epigenetics. 2016;8:114.

28. Amarasekera M, Noakes $P$, Strickland D, Saffery R, Martino DJ, Prescott SL. Epigenome-wide analysis of neonatal CD4(+) T-cell DNA methylation sites potentially affected by maternal fish oil supplementation. Epigenetics. 2014; 9(12):1570-6.

29. Hoile SP, Irvine NA, Kelsall CJ, Sibbons C, Feunteun A, Collister A, et al. Maternal fat intake in rats alters 20:4n-6 and 22:6n-3 status and the epigenetic regulation of Fads2 in offspring liver. J Nutr Biochem. 2013;24(7): 1213-20.

30. Chisaguano AM, Montes R, Pérez-Berezo T, Castellote Al, Guerendiain M, Bustamante $M$, et al. Gene expression of desaturase (FADS1 and FADS2) and Elongase (ELOVL5) enzymes in peripheral blood: association with polyunsaturated fatty acid levels and atopic eczema in 4-year-old children. PLoS One. 2013;8(10):e78245.

31. Nwaru BI, Erkkola M, Lumia M, Kronberg-Kippila C, Ahonen S, Kaila M, et al. Maternal intake of fatty acids during pregnancy and allergies in the offspring. Br J Nutr. 2012;108(4):720-32.

32. Arshad SH, Holloway JW, Karmaus W, Zhang H, Ewart S, Mansfield L, et al. Cohort profile: the Isle of Wight whole population birth cohort (IOWBC). Int J Epidemiol. 2018;47(4):1043-i.

33. Arshad SH, Karmaus W, Zhang H, Holloway JW. Multigenerational cohorts in patients with asthma and allergy. J Allergy Clin Immunol. 2017;139(2):415-21.

34. Arshad SH, Karmaus W, Kurukulaaratchy R, Sadeghnejad A, Huebner M, Ewart S. Polymorphisms in the interleukin 13 and GATA binding protein 3 genes and the development of eczema during childhood. $\mathrm{Br} J$ Dermatol. 2008;158(6):1315-22.

35. Hanifin JM. Atopic dermatitis. J Am Acad Dermatol. 1982;6(1):1-13.

36. Venter C, Higgins B, Grundy J, Clayton CB, Gant C, Dean T. Reliability and validity of a maternal food frequency questionnaire designed to estimate consumption of common food allergens. J Hum Nutr Diet. 2006;19(2):129-38.

37. Arterburn LM, Hall EB, Oken H. Distribution, interconversion, and dose response of n-3 fatty acids in humans. Am J Clin Nutr. 2006;83(6 Suppl): 1467S-76S.

38. Howie B, Fuchsberger C, Stephens M, Marchini J, Abecasis GR. Fast and accurate genotype imputation in genome-wide association studies through pre-phasing. Nat Genet. 2012;44(8):955-9.

39. Miller SA, Dykes DD, Polesky HF. A simple salting out procedure for extracting DNA from human nucleated cells. Nucleic Acids Res. 1988;16(3):1215.

40. Bibikova M, Fan JB. GoldenGate assay for DNA methylation profiling. Methods Mol Biol. 2009:507:149-63.

41. Lehne B, Drong AW, Loh M, Zhang W, Scott WR, Tan ST, et al. A coherent approach for analysis of the Illumina HumanMethylation450 BeadChip improves data quality and performance in epigenome-wide association studies. Genome Biol. 2015;16:37.

42. Johnson WE, Li C, Rabinovic A. Adjusting batch effects in microarray expression data using empirical Bayes methods. Biostatistics. 2007;8(1):118-27.

43. Ritchie ME, Phipson B, Wu D, Hu Y, Law CW, Shi W, et al. limma powers differential expression analyses for RNA-sequencing and microarray studies. Nucleic Acids Res. 2015;43(7):e47.

44. Ritchie ME, Silver J, Oshlack A, Holmes M, Diyagama D, Holloway A, et al. A comparison of background correction methods for two-colour microarrays. Bioinformatics. 2007;23(20):2700-7.

45. Rothman KJ. No adjustments are needed for multiple comparisons. Epidemiology. 1990;1(1):43-6.

\section{Publisher's Note}

Springer Nature remains neutral with regard to jurisdictional claims in published maps and institutional affiliations. 